\title{
Adherence to quality measures improves survival in esophageal cancer in a retrospective cohort study of the national cancer database from 2004 to 2016
}

\author{
Akash Adhia $^{1}$, Joseph Feinglass ${ }^{1,2}$, Cary Jo Schlick ${ }^{1,3,4}$, David Odell ${ }^{1,3,4,5}$ \\ ${ }^{1}$ Surgical Outcomes and Quality Improvement Center, Department of Surgery, Northwestern University, Feinberg School of Medicine, Chicago, \\ IL, USA; ${ }^{2}$ Department of Medicine, Northwestern University, Feinberg School of Medicine, Chicago, IL, USA; ${ }^{3}$ Northwestern Institute for \\ Comparative Effectiveness Research in Oncology (NICER-Onc), Robert H. Lurie Comprehensive Cancer Center, Northwestern University, \\ Feinberg School of Medicine, Chicago, IL, USA; ${ }^{4}$ Center for Healthcare Studies, Institute for Public Health and Medicine, Northwestern University, \\ Feinberg School of Medicine, Chicago, IL, USA; ${ }^{5}$ Division of Thoracic Surgery, Department of Surgery, Northwestern University, Feinberg School \\ of Medicine, Chicago, IL, USA \\ Contributions: (I) Conception and design: All authors; (II) Administrative support: All authors; (III) Provision of study materials or patients: All \\ authors; (IV) Collection and assembly of data: All authors; (V) Data analysis and interpretation: All authors; (VI) Manuscript writing: All authors; (VII) \\ Final approval of manuscript: All authors. \\ Correspondence to: David Odell, MD, MMSc. Surgical Outcomes and Quality Improvement Center, 633 N. St. Clair Street; 20th floor, Chicago, IL \\ 60611, USA. Email: dodell@nm.org.
}

Background: We assessed adherence to four novel quality measures in patients with stage III esophageal cancer, a leading cause of death among GI malignancies.

Methods: We performed a retrospective cohort study of 22,871 stage III esophageal cancer patients identified from the National Cancer Database (NCDB) between 2004 and 2016. Four quality measures were defined from published guidelines: administration of induction therapy, $>15$ lymph nodes sampled, surgery within 60 days of neoadjuvant treatment, and R0 resection. The association of patient demographic and treatment variables with measure adherence was assessed using multiple logistic regression. Risk of all-cause mortality was assessed comparing adherent and non-adherent cases using Cox modeling. Kaplan-Meier survival estimates of groups that adhered to zero to four out of four quality measures were performed.

Results: Adherence was high for neoadjuvant treatment (93.7\%), timing of surgery (85.7\%) and completeness of resection (92.0\%), but low for nodal evaluation (45.9\%). Medicaid insurance status was associated with decreased odds of adherence for neoadjuvant treatment [odds ratio (OR) $0.73,95 \%$ confidence interval (CI): 0.54-0.99], nodal evaluation (OR 0.81, 95\% CI: 0.68-0.96), and completeness of resection (OR 0.71, 95\% CI: 0.54-0.92). From 2010 to 2016, when compared to cases from 2004 to 2005, there was a progressive increase in the odds of adequate induction therapy, nodal staging, and completeness of resection, but a progressive decrease in odds of well-timed surgery. Adherence was associated with decreased all-cause mortality for induction therapy, nodal staging, and R0 resection, but not for timing of surgery. Survival improved as the number of quality measures an individual patient adhered to increased.

Conclusions: Adherence to quality measures is associated with improved survival in patients with stage III esophageal cancer. Understanding variability in measure adherence may identify targets for quality improvement initiatives.

Keywords: Esophageal neoplasms; database; survival; esophagectomy

Submitted May 19, 2020. Accepted for publication Aug 05, 2020.

doi: $10.21037 /$ jtd-20-1347

View this article at: http://dx.doi.org/10.21037/jtd-20-1347

(c) Journal of Thoracic Disease. All rights reserved. 


\section{Introduction}

Esophageal cancer is the most lethal of GI malignancies with an estimated incidence of 17,650 and 16,080 deaths in 2019 (1). High quality esophageal cancer care demands accurate and timely staging, multidisciplinary cooperation, and prompt delivery of treatment (2-11). To make delivery of care more complicated, patients frequently have minimal signs and symptoms of malignancy until it has progressed substantially, contributing to challenges in treatment decision-making. A classic patient presents with dysphagia usually secondary to at least a stage III cancer.

Stage IV cancer, unfortunately, has poor prognosis despite treatment and is typically treated with palliative and not curative intent (11). On the other hand, several studies have shown that certain treatment regimens can improve outcomes in stage III cancer $(12,13)$. These studies have been distilled into trusted guidelines like the National Comprehensive Cancer Network (NCCN) guidelines, Society of Thoracic Surgeons (STS) guidelines and others (2-11). While these guidelines help providers determine management every day, we have limited insight into whether we are following guidelines nationally. Previous studies have shown national variability in treatment in gynecologic, otolaryngologic, skin and rectal cancer (14-18). For that reason, we suspect similar deficits in guideline concordant care exist for esophageal cancer as well. Studying the extent and drivers of divergence from guidelines would provide direction for quality improvement, an opportunity to improve outcomes for patients who suffer from this deadly disease.

Given the lethality of esophageal cancer, its typical late presentation, and the opportunity to increase life expectancy in stage III cancer with the appropriate treatment regimen, it is vital that these patients receive high quality care. The first step in developing quality improvement initiatives is characterizing areas of strength and improvement. While we have numerous guidelines published by the aforementioned societies, we currently lack published quality measures in esophageal cancer-statements that clearly define inclusion criteria and adherence thresholds-to effectively determine the rate of guideline concordant care. In this study, we built four novel quality measures from published guidelines and then evaluated their viability by measuring their association with survival and national adherence rates. A similar study was performed by Samson et al., who showed variability in quality measure adherence and found a correlation between adherence to quality measures in esophagectomy and survival from 1998 to 2012 in the National Cancer Database
(NCDB) (19). To build on their research, we performed a more recent analysis from 2004 to 2016 in the NCDB and evaluated additional aspects of esophageal cancer care. We also evaluated additional demographic and treatment explanatory variables. We evaluated two critical aspects of esophageal cancer care-multidisciplinary coordination and surgical completeness-by assessing adherence to four novel quality measures. Then, we investigated all-cause mortality when a patient's care is adherent to each quality measure and adherent to an increasing number of quality measures. We present the following article in accordance with the STROBE reporting checklist (available at http:// dx.doi.org/10.21037/jtd-20-1347).

\section{Methods}

\section{Data source}

In this retrospective cohort study, patients diagnosed with stage III esophageal cancer were identified from the NCDB from 2004 to 2016. The NCDB characterizes diagnosis, staging, and treatment for roughly $70 \%$ of new cancer diagnoses, including all those at hospitals accredited by the American Cancer Society and the Commission on Cancer of the American College of Surgeons.

\section{Patients}

Patients were included in the study if they were greater than 18 years old and had clinical stage III disease. Additionally, to avoid patients with incomplete or ambiguous reporting in the NCDB, patients were only included if they had only one instance of cancer, the reporting facility performed or determined the course of treatment, and the diagnosis occurred on or after the facility was required to report to the NCDB.

\section{Measures}

The following four novel quality measures were derived from existing guidelines and studies (2-11) because they were easily quantifiable and binary determination of compliance (either adherent or non-adherent) was possible from pre-existing NCDB data:

(I) Induction therapy: IF a patient has a clinical stage 3 esophageal cancer, THEN induction chemotherapy and/or radiation should be performed before surgery;

(II) Nodal staging: IF a patient has a clinical stage 3 
esophageal cancer, THEN at least 15 lymph nodes should be removed;

(III) Timing of surgery: IF a patient receives surgery and chemotherapy and/or radiation, THEN the surgery should be performed no more than sixty days after the completion of induction therapy;

(IV) Completeness of resection: IF a patient has surgery, THEN they should receive an R0 resection.

Each quality measure had its own analytical group because each had unique inclusion criteria and consequently was analyzed independently from the others. For induction therapy, patients were included in the analytical group if chemotherapy and/or radiation was performed and excluded if intraoperative therapy was performed or the data did not exist. Adherent cases must have received preoperative chemotherapy and/or radiation therapy and may have also received postoperative chemoradiation. For lymph node sampling, patients were included if the number of lymph nodes sampled was reported and there was some surgery performed. Adherent cases had at least 15 lymph nodes examined. For timing of surgery, patients were included if a surgery was performed and radiation and/or chemotherapy were administered before surgery. The NCDB is missing data to directly evaluate if surgery was performed within 60 days of the completion of chemotherapy. For that reason, cases with adequate timing of surgery had surgery performed no more than sixty days after the completion of radiation or no more than four months from the beginning of chemotherapy. If a course of chemotherapy takes about eight weeks, this estimates that surgery occurs within 60 days. Adherent cases had either surgery within 60 days of the end of radiation and/or within 120 days of the beginning of chemotherapy. For completeness of resection, patients were included if they had surgical margins data reported. Adherent cases had an R0 resection.

\section{Patient and hospital characteristics}

Patient characteristics included sex, age stratified into tenyear brackets, race and ethnicity, zip code income quartiles, zip code education quartiles, insurance status dichotomized to "Medicaid/uninsured" or "other", region type (large urban, medium urban, small urban, rural, and unknown), institution type dichotomized to "academic" or "nonacademic", location of lesion [middle third, lower third, overlapping, or esophagus not otherwise specified (NOS)], histology [squamous cell carcinoma (SCC), adenocarcinoma (AC), or other], and date of diagnosis stratified into five two-year periods form 2004 to 2016. More information on these variables and groupings can be found in the NCDB Participant User Files Data Dictionary (20). The number and percent of these characteristics in the overall group and each analytical group were calculated.

\section{Statistical analysis}

The primary outcome of adherence was calculated as a percentage of each analytical group adherent to its quality measure. A patient's care was judged in binary fashion, either adherent or not adherent. Patients with any missing data were excluded from analysis. The significance of differences in adherence between patient demographic, hospital, time, and treatment variables was assessed using bivariate chi-square tests and multiple logistical regression adjusted for demographic, clinical, hospital and time covariates. The significance of differences in time to all-cause mortality between patients who experienced increasing levels of adherent care (between groups that adhered to none, one of four, two of four etc. quality measures) was determined by log rank tests from KaplanMeier survival probabilities. Hazard ratios for the risk of the secondary outcome of all-cause mortality were computed by fitting four Cox proportional hazards models-one for each quality measure-which controlled for covariates. Survival analyses were censored at last follow-up. All multivariate analyses had standard errors adjusted for clustering of observations within hospitals. A significance level of $\mathrm{P}<0.05$ was used. STATA version 15 (College Station, Texas, US) was used for all analyses.

\section{IRB approval}

The study was conducted in accordance with the Declaration of Helsinki (as revised in 2013). This study of deidentified, public data was ruled exempt by the Northwestern University Institutional Review Board and informed consent was not required as it is defined by the United States Department of Health and Human Services as secondary research of biospecimens that are publicly available (21).

\section{Results}

Of 154,589 esophageal cancer cases in the NCDB, these selection criteria yielded 22,871 patients diagnosed with stage III esophageal cancer (Figure 1). 


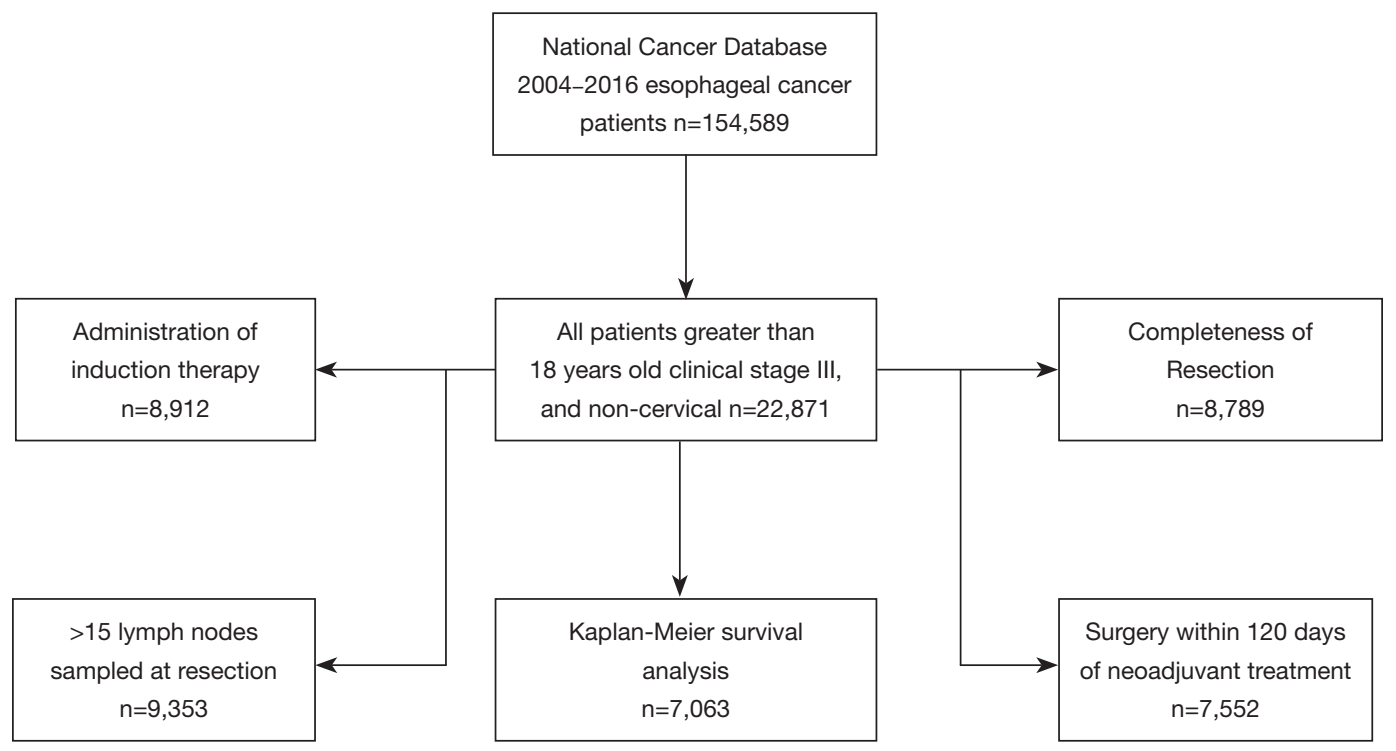

Figure 1 Consort diagram showing number of patients in the overall sample, general selection, selection for each quality measure and selection for Kaplan-Meier survival analysis.

\section{Baseline demographics}

The patients included in this study were mostly male with peak age bracket of $60-69$. Only about $10 \%$ were under 50 years old. Most (79.6\%) were non-Hispanic whites. There were slightly greater proportion of quartile 3 or 4 patients than quartile 1 and 2 for education (54.6\%) and income $(58.0 \%)$. Most patients were non-Medicaid and non-uninsured. About half of patients were treated at an academic medical center (46.6\%). There were more cases of adenocarcinoma $(61.0 \%)$ than squamous cell carcinoma (27.9\%). There were progressively more cases from 2004 to 2016 (Table 1).

\section{Measure adberence}

For each quality measure, there was an adequate amount of data for the following number of patients: 8,912 for induction therapy; 9,353 cases for lymph node sampling; 7,552 cases for timing of surgery; 8,789 cases for completeness of resection (Figure 1). Adherence was high for induction therapy (93.7\%), timing of surgery (85.7\%), and completeness of resection (92.0\%) and only adequate in a minority of patients for nodal staging (45.9\%) (Figure 2).

\section{Induction therapy}

With respect to induction therapy, all chi-square univariate analyses yielded significance $(\mathrm{P}<0.05)$ except for education quartile, income quartile and region type. Cases were more likely to have adherent induction therapy if they were male rather than female [odds ratio (OR) 1.35, 95\% CI: 1.09-1.68], at an academic institution as opposed to a nonacademic one (OR 1.34, 95\% CI: 1.03-1.73), and have SCC (OR 1.35, 95\% CI: 1.03-1.78, REF: AC). When compared to those that were treated from 2004 to 2005, there was a progressive increase in odds of adherence from 2008 to 2009 (OR 1.59, 1.11-2.30) to 2014 to 2016 (OR 2.95, 95\% CI: 2.10-4.14). They were less likely to be adherent if they were 70 to 79 years old $(0.64,95 \%$ CI: $0.50-0.82$, REF: 60 to 69 years old), $80+($ OR $0.19,95 \%$ CI: $0.12-0.29)$, non-Hispanic blacks (0.62, 95\% CI: 0.43-0.89, REF: nonHispanic whites)], or Medicaid or uninsured patients (OR 0.73, 95\% CI: 0.54-0.99) (Table 2).

\section{Nodal staging}

For nodal staging, all univariate analyses were significant except for age and region type. Like induction therapy, cases were more likely to have adherent nodal staging if they at an academic institution (OR 1.75, 95\% CI: 1.42-2.16) and there was a progressive increase in odds of adherence from 2006 to 2007 (OR 1.32, 95\% CI: 1.06-1.63) to 2014-2016 (OR 3.44, 95\% CI: 2.64-4.50) when compared to 2004-2005. They were less likely to be adherent if they 
Table 1 Percent of patients eligible for each quality measure and of the general selection

\begin{tabular}{|c|c|c|c|c|c|c|c|c|c|c|}
\hline \multirow[t]{2}{*}{ Characteristics } & \multicolumn{2}{|c|}{$\begin{array}{l}\text { Induction therapy } \\
\qquad(\mathrm{n}=8,912)\end{array}$} & \multicolumn{2}{|c|}{$\begin{array}{l}\text { Nodal Staging } \\
\qquad(\mathrm{n}=9,353)\end{array}$} & \multicolumn{2}{|c|}{$\begin{array}{l}\text { Timing of surgery } \\
\qquad(n=7,552)\end{array}$} & \multicolumn{2}{|c|}{$\begin{array}{l}\text { Completeness of resection } \\
\qquad(\mathrm{n}=8,789)\end{array}$} & \multicolumn{2}{|c|}{$\begin{array}{l}\text { General selection } \\
\qquad(n=22,871)\end{array}$} \\
\hline & $\mathrm{N}$ & $\%$ & $\mathrm{~N}$ & $\%$ & $\mathrm{~N}$ & $\%$ & $\mathrm{~N}$ & $\%$ & $\mathrm{~N}$ & $\%$ \\
\hline Female & 1,347 & 15.11 & 1,471 & 15.73 & 1,135 & 15.03 & 1,335 & 15.19 & 4,495 & 19.65 \\
\hline $18-29$ & 19 & 0.21 & 18 & 0.19 & 16 & 0.21 & 18 & 0.20 & 41 & 0.18 \\
\hline $30-39$ & 150 & 1.68 & 148 & 1.58 & 116 & 1.54 & 145 & 1.65 & 246 & 1.08 \\
\hline $40-49$ & 845 & 9.48 & 843 & 9.01 & 716 & 9.48 & 814 & 9.26 & 1,669 & 7.30 \\
\hline $80+$ & 139 & 1.56 & 226 & 2.42 & 99 & 1.31 & 165 & 1.88 & 2,059 & 9.00 \\
\hline \multicolumn{11}{|l|}{$\begin{array}{l}\text { Race and } \\
\text { ethnicity }\end{array}$} \\
\hline $\begin{array}{l}\text { Non-Hispanic } \\
\text { White }\end{array}$ & 7,755 & 87.02 & 8,098 & 86.58 & 6,618 & 87.63 & 7,647 & 87.01 & 18,199 & 79.57 \\
\hline $\begin{array}{l}\text { Non-Hispanic } \\
\text { Black }\end{array}$ & 324 & 3.64 & 371 & 3.97 & 247 & 3.27 & 308 & 3.50 & 2,091 & 9.14 \\
\hline Hispanic & 613 & 6.88 & 647 & 6.92 & 500 & 6.62 & 615 & 7.00 & 1,863 & 8.15 \\
\hline Second quartile & 2,010 & 22.55 & 2,121 & 22.68 & 1,711 & 22.66 & 1,982 & 22.55 & 5,205 & 22.76 \\
\hline Third quartile & 2,348 & 26.35 & 2,459 & 26.29 & 1,991 & 26.36 & 2,327 & 26.48 & 5,857 & 25.61 \\
\hline Highest quartile & 3,215 & 36.07 & 3,317 & 35.46 & 2,745 & 36.35 & 3,148 & 35.82 & 7,411 & 32.40 \\
\hline Other/unknown & 99 & 1.11 & 100 & 1.07 & 81 & 1.07 & 91 & 1.04 & 285 & 1.25 \\
\hline \multicolumn{11}{|l|}{$\begin{array}{l}\text { Zip code } \\
\text { education quartile }\end{array}$} \\
\hline Lowest quartile & 1,184 & 13.29 & 1,274 & 13.62 & 997 & 13.20 & 1,181 & 13.44 & 4,090 & 17.88 \\
\hline Second quartile & 2,289 & 25.68 & 2,415 & 25.82 & 1,917 & 25.38 & 2,249 & 25.59 & 6,035 & 26.39 \\
\hline Third quartile & 2,893 & 32.46 & 3,009 & 32.17 & 2,458 & 32.55 & 2,839 & 32.30 & 6,932 & 30.31 \\
\hline Highest quartile & 2,455 & 27.55 & 2,563 & 27.40 & 2,105 & 27.87 & 2,436 & 27.72 & 5,555 & 24.29 \\
\hline Other/unknown & 91 & 1.02 & 92 & 0.98 & 75 & 0.99 & 84 & 0.96 & 259 & 1.13 \\
\hline
\end{tabular}

Table 1 (continued) 
Table 1 (continued)

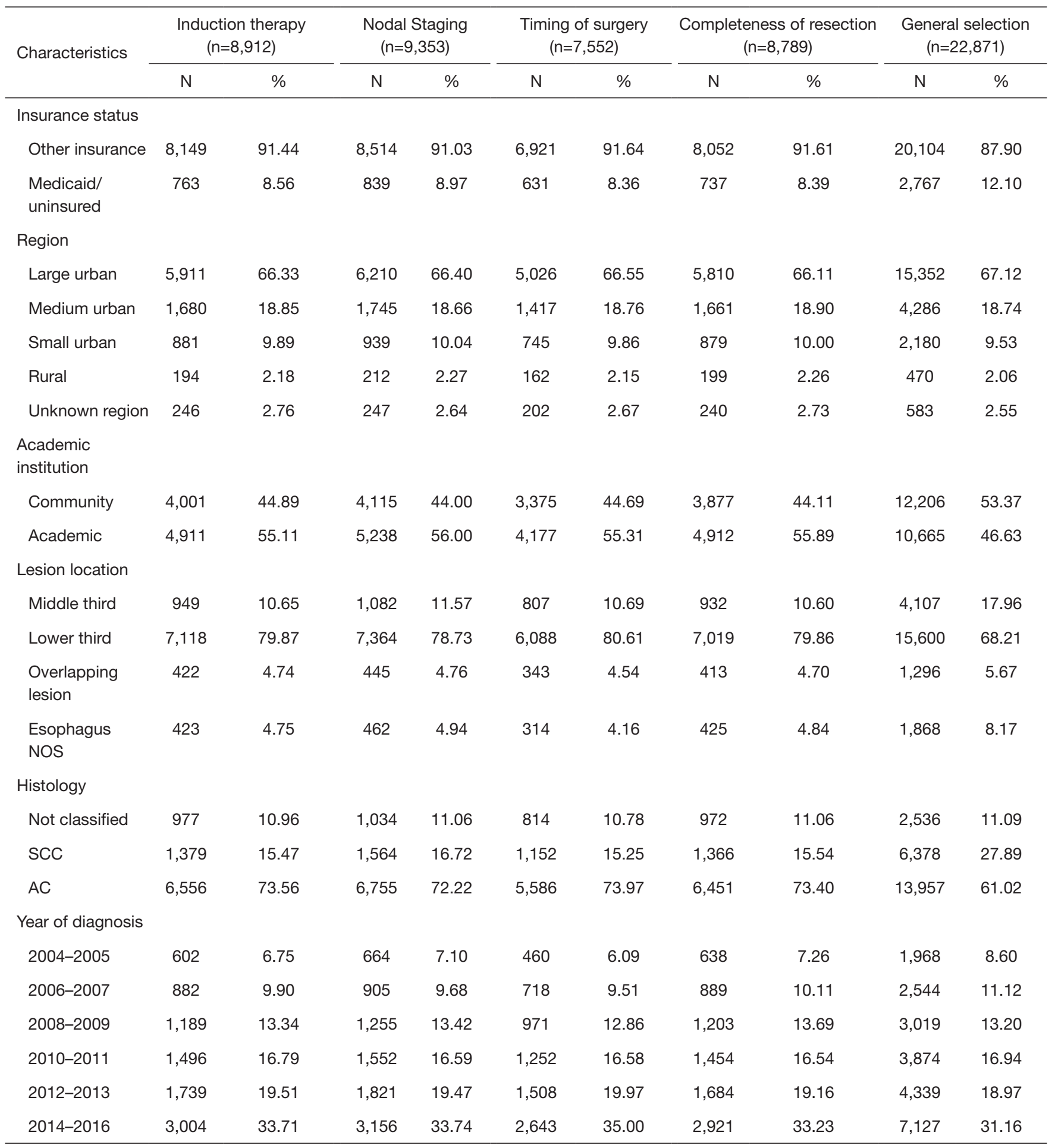




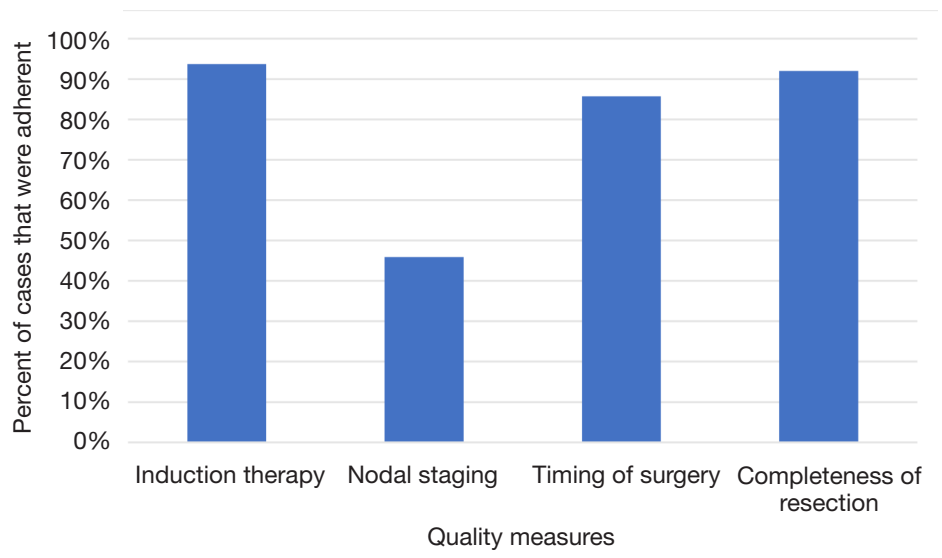

Figure 2 Adherence rates for each quality measure.

were Medicaid patients or uninsured (OR 0.81, 95\% CI: 0.68-0.96) (Table 2).

\section{Timing of surgery}

For timing of surgery, all univariate analyses were significant except for income quartile, education quartile, academic institution, and lesion location. Patients were more likely to be adherent to timing of surgery if they were from medium urban region (OR 1.30, 95\% CI: 1.06-1.60) or rural geographic region (OR 1.85, 95\% CI: 1.05-3.26) when compared to those from a large urban region. They were less likely to be adherent if they were in the third income quartile (OR 0.76, 95\% CI: 0.63-0.91). Diverging from the pattern seen in the other quality measures, there was a progressive decrease in the odds of timely surgery from 2010-2011 (OR 1.43, 95\% CI: $1.03-1.97)$ to 2014-2016 (OR 1.86, 95\% CI: 1.35-2.56) when compared patients treated in 2004-2005 (Table 2).

\section{Completeness of resection}

With respect to completeness of resection, all univariate analyses were significant except for sex. Cases were more likely to receive complete resections if they were treated at an academic institution (OR 1.42, 95\% CI: 1.19-1.69) and, like induction therapy and nodal resection, there was a progressive increase in odds adherence from 2010 to 2011 (OR 1.43, 95\% CI: 1.03-1.97) to 2014-2016 (OR 1.86, 95\% CI: $1.35-2.56)$ when compared to 2004-2005. They were less likely to be adherent if they were greater than 80 years old (OR 0.33, 95\% CI: 0.21-0.51), Medicaid or uninsured (OR 0.71, 95\% CI: 0.54-0.92), if the lesion was in the middle third of the esophagus (OR 0.66, 95\% CI: $0.51-0.87)$, and if the lesion overlapped from middle to lower esophagus (OR 0.59, 95\% CI: 0.43-0.82) (Table 2).

\section{Survival models}

Achieving adherence was associated with a statistically significant decrease in all-cause mortality for administration of induction therapy (HR $0.67,95 \%$ CI: $0.60-0.74$ ), nodal staging (HR 0.94, 95\% CI: 0.88-0.998), and R0 resection (HR $0.51,95 \%$ CI: $0.47-0.57$ ), but not for timing of surgery (HR 0.95 , 95\% CI: 0.86-1.04). Survival improved as the number of quality measures an individual patient adhered to increased (Figure 3) and was shown to be significant by logrank testing $(\mathrm{P}<0.001)$. The 5 -year survival rate was $7.5 \%$ (95\% CI: $1.7-19.4 \%$ ) for those adherents to one quality measure, $28.0 \%(24.5-31.6 \%)$ for those adherents to two quality measures, $31.9 \%(30.2-33.7 \%)$ for those adherents to three, and $36.9 \%(34.8-39.1 \%)$ for those adherents to four out of four quality measures. The median followup time of last contact or death was 14.5 months with an interquartile range of 23.0 months for all patients that met the general inclusion criteria $(\mathrm{n}=22,871$, Figure 1).

\section{Discussion}

Stage III esophageal cancer is a deadly disease whose management demands high surgical performance outcomes and timely multidisciplinary coordination. Consequently, both process and outcome measures were included in this analysis of esophageal cancer care. To investigate variability in treatment, our objective was to assess adherence to four novel process and outcome quality measures in patients 
Table 2 Odds ratios of characteristics as predictors of adherence to the four quality measures

\begin{tabular}{|c|c|c|c|c|}
\hline Characteristics $^{\dagger}$ & Induction therapy & Nodal staging & Timing of surgery & Completeness of resection \\
\hline Male & $1.35(1.09-1.68)^{\star}$ & $1.12(0.99-1.26)$ & $1.23(1.04-1.45)^{\star}$ & $0.98(0.79-1.23)$ \\
\hline Female & Ref & Ref & Ref & Ref \\
\hline \multicolumn{5}{|l|}{ Age, years } \\
\hline 30-39 & $0.94(0.50-1.78)$ & $1.28(0.88-1.84)$ & $1.79(0.97-3.31)$ & $0.77(0.46-1.28)$ \\
\hline $40-49$ & $1.06(0.75-1.50)$ & $1.08(0.92-1.27)$ & $1.20(0.95-1.51)$ & $1.00(0.75-1.33)$ \\
\hline $50-59$ & $0.94(0.75-1.18)$ & $1.03(0.93-1.13)$ & $1.11(0.96-1.29)$ & $0.91(0.76-1.10)$ \\
\hline $60-69$ & Ref & Ref & Ref & Ref \\
\hline \multicolumn{5}{|l|}{ Race } \\
\hline Non-Hispanic white & Ref & Ref & Ref & Ref \\
\hline Non-Hispanic black & $0.62(0.43-0.89)^{\star}$ & $0.79(0.62-1.02)$ & $0.74(0.52-1.05)$ & $0.87(0.56-1.33)$ \\
\hline Hispanic & $0.81(0.59-1.11)$ & $1.01(0.79-1.28)$ & $0.95(0.72-1.24)$ & $0.75(0.57-0.98)^{*}$ \\
\hline Asian & $4.39(1.06-18.19)^{\star}$ & $1.20(0.83-1.74)$ & $1.07(0.66-1.75)$ & $0.96(0.49-1.89)$ \\
\hline Other or unknown & $0.99(0.40-2.43)$ & $1.38(0.87-2.20)$ & $0.86(0.41-1.79)$ & $1.48(0.62-3.52)$ \\
\hline \multicolumn{5}{|l|}{ Income quartile } \\
\hline Lowest quartile & $1.03(0.66-1.61)$ & $0.94(0.73-1.22)$ & $0.88(0.65-1.21)$ & $0.83(0.58-1.18)$ \\
\hline Lowest quartile & $0.92(0.61-1.37)$ & $0.77(0.60-1.00)$ & $0.81(0.60-1.10)$ & $0.90(0.65-1.24)$ \\
\hline Second quartile & $0.86(0.64-1.17)$ & $0.93(0.78-1.10)$ & $0.94(0.75-1.17)$ & $0.96(0.75-1.22)$ \\
\hline Third quartile & $0.89(0.69-1.15)$ & $1.02(0.90-1.16)$ & $0.99(0.81-1.21)$ & $1.10(0.89-1.37)$ \\
\hline Highest quartile & Ref & Ref & Ref & Ref \\
\hline \multicolumn{5}{|l|}{ Insurance status } \\
\hline Other insurance & Ref & Ref & Ref & Ref \\
\hline Medicaid or uninsured & $0.73(0.54-0.99)^{\star}$ & $0.81(0.68-0.96)^{\star}$ & $0.79(0.62-1.00)$ & $0.71(0.54-0.92)^{*}$ \\
\hline
\end{tabular}

Table 2 (continued) 
Table 2 (continued)

\begin{tabular}{|c|c|c|c|c|}
\hline Characteristics & Induction therapy & Nodal staging & Timing of surgery & Completeness of resection \\
\hline \multicolumn{5}{|l|}{ Region type } \\
\hline Large urban & Ref & Ref & Ref & Ref \\
\hline Medium urban & $0.98(0.74-1.30)$ & $0.91(0.78-1.06)$ & $1.30(1.06-1.60)^{\star}$ & $0.84(0.68-1.04)$ \\
\hline Small urban & $1.02(0.74-1.40)$ & $0.99(0.78-1.25)$ & $1.32(0.98-1.77)$ & $1.01(0.75-1.36)$ \\
\hline Unknown region & $0.94(0.58-1.51)$ & $1.11(0.82-1.50)$ & $0.86(0.58-1.28)$ & $1.02(0.62-1.68)$ \\
\hline \multicolumn{5}{|l|}{ Institution type } \\
\hline Academic & $1.34(1.03-1.73)^{\star}$ & $1.75(1.42-2.16)^{\star}$ & $0.99(0.80-1.23)$ & $1.42(1.19-1.69)^{\star}$ \\
\hline Non-academic & Ref & Ref & Ref & Ref \\
\hline Lower third & Ref & Ref & Ref & Ref \\
\hline Overlapping lesion & $0.74(0.50-1.10)$ & $1.12(0.91-1.38)$ & $1.00(0.74-1.35)$ & $0.59(0.43-0.82)^{\star}$ \\
\hline Esophagus (not specified) & $0.39(0.28-0.54)^{\star}$ & $0.75(0.60-0.93)^{\star}$ & $0.94(0.67-1.32)$ & $0.71(0.49-1.01)$ \\
\hline Other & $1.03(0.74-1.43)$ & $1.20(0.99-1.44)$ & $1.11(0.83-1.48)$ & $0.41(0.30-0.56)^{\star}$ \\
\hline \multicolumn{5}{|l|}{ Histology } \\
\hline Adenocarcinoma & Ref & Ref & Ref & Ref \\
\hline Squamous cell carcinoma & $1.35(1.03-1.78)^{\star}$ & $1.09(0.94-1.26)$ & $1.13(0.92-1.38)$ & $0.91(0.70-1.18)$ \\
\hline \multicolumn{5}{|l|}{ Date of case } \\
\hline 2014-2016 & $2.95(2.10-4.14)^{\star}$ & $3.44(2.64-4.50)^{*}$ & $0.45(0.30-0.68)^{\star}$ & $1.86(1.35-2.56)^{\star}$ \\
\hline
\end{tabular}

${ }^{*}, \mathrm{P}<0.05{ }^{\dagger}$, multivariate logistic regression predictors (OR and $95 \% \mathrm{Cl}$ ).

with stage 3 esophageal cancer and to investigate survival as patients receive increasingly adherent care. Doing so would provide direction for future quality improvement initiatives.

Donabedian's framework for quality improvement and delivery of care shows how these quality measures fit into evaluating treatment of esophageal cancer. Structure (the environment in which care is given like a high-frequency academic center or community hospital) sets the stage for processes, (how the care is delivered and which order) which ultimately lead to outcomes-like time to all-cause mortality or rehospitalization (22). In this current study, induction therapy, timing of surgery, and nodal staging are all process measures because they can be measured as care is delivered. On the other hand, although completeness of resection describes a surgeon's actions, it can only be measured by microscopy after the surgery. It is therefore an outcome measure. This study does not evaluate any structural aspects of esophageal cancer care, a potential future investigation.

These specific quality measures were derived from existing guidelines and studies because they were easily quantifiable with binary determination of compliance 
being possible from pre-existing NCDB data. In other words, care was measured as a dichotomous "adherent" or "not adherent"; there is no spectrum of adherence within a single quality measure. Moreover, we adjusted them to have the fewest, most clear, and most generous inclusion criteria possible to maximize generalizability and ease of implementation in clinic while still maintaining the essential structure and legitimacy of the root guideline. The survival models show that despite adjustments to accepted guidelines, these novel quality measures remain useful and still evaluate practices that improve time to allcause mortality. Simplicity of inclusion criteria and binary determination of adherence make these quality measures strong candidates for use in everyday clinical quality evaluation.

\section{Multidisciplinary care coordination}

Induction therapy and timing of surgery evaluate the effectiveness of multidisciplinary care since both medical and surgical oncology teams must coordinate to make sure that the patient receives therapy in the appropriate order and at the appropriate time: induction chemotherapy and/ or radiation then surgery within 60 days. Adherence was particularly high in both these measures indicating that multidisciplinary coordination is a strength of esophageal cancer care nationally. Our 2004 to 2016 adherence rate (93.7\%) concurs with the Samson et al. 2006 to 2012 analysis of NCDB data, where they show $85.8 \%$ adherence to induction therapy; they did not analyze timing of surgery (19). In addition, we show that induction therapy prolongs time to all-cause mortality, making a case to maintain strong adherence to this quality measure nationally. The quality measure for timing of surgery should be reevaluated because appropriate timing did not show a significant survival benefit.

We developed our induction therapy quality measure from guidelines $(2,4)$ that cite the CROSS trial, modifying the inclusion as well as adherence criteria. van Hagen et al. selected patients that have lesions that are $3 \mathrm{~cm}$ below the upper esophageal sphincter, length and width not exceeding 8 and $5 \mathrm{~cm}$ respectively, stage 3 , and patients between 18-75 years of age. We selected for patients similarly except that we did not limit patients based on the size of the tumor. Furthermore, we expanded adherence patient care to include chemotherapy and/or radiotherapy, not necessarily both as in the CROSS trial. These adjustments might call into question the strength of this quality measure. Despite this discrepancy, however, we show that induction therapy provided an overall survival benefit when compared to those who did not receive adequate induction therapy, indicating this quality measure's usefulness and legitimacy.

There are limitations to analysis in the NCDB for induction therapy. A limitation of the NCDB is that there is not adequate differentiation between patients who did not receive chemotherapy for a legitimate reason or refusal and those that were perfectly acceptable candidates but were not offered the gold standard care. For that reason, adherence to this quality measure might be much lower if we were able to include the patients who had no induction chemotherapy offered but were acceptable candidates for standard treatment. Another area of ambiguity is cases where chemotherapy and/or radiation was administered between two surgical procedures. We deemed these cases as adherent because it is possible that the first procedure was exploratory or was solely for lymph node resection and the second with curative intent (esophagectomy). If the first surgical procedure had curative intent, however, then these cases would be inappropriately deemed adherent because our quality measure dictates that induction therapy should always be before esophagectomy, falsely raising the adherence level for this quality measure.

There are varied conclusions from previous studies on the ideal timing of surgery after esophagectomy (13,23-26). We chose 60 days as a maximum appropriate time between chemotherapy and/or radiation and surgery following evidence from another NCDB study by Franko et al. (13). We preferred the results of that study over others because the data source, NCDB, and study setup, retrospective, matched our study. Our analysis suggests, however, that there is no survival benefit to performing surgery before 60 days. Possible sources of discrepancy between Franko's study and ours are that all of Franko et al.'s patients received both chemotherapy and radiation, while ours received either or both. Furthermore, all histologic subtypes were included in our study while Franko et al. limited its analytical group to just adenocarcinoma. The lack of survival benefit calls into question the usefulness of this quality measure as it is currently structured. There is probably potential for a more effective measure as this is the only one of our four quality measures with no significant survival benefit.

There are also NCDB-related limitations for evaluating timing of surgery. There exists adequate data to calculate days from the end of radiation to surgery, but not for chemotherapy. Chemotherapy treatment was assumed to be 8 weeks long (about 60 days) and time from the start 
of chemotherapy to the definitive surgical procedure was calculated, adherent cases having less than or equal to 120 days. This estimation would underestimate adherence for cases where chemotherapy regimens lasted longer than 60 days and overestimate adherence for cases where chemotherapy regimens were shorter than 60 days.

\section{Surgical completeness}

Nodal staging and completeness of resection are both measures of surgical completeness. They depend only on the surgeon and clinical coordination factors are not involved. Adequate nodal staging is essential because it guides clinicians to the most efficacious treatment. Unfortunately, this study shows that nodal staging is highly variable nationally $(45.9 \%)$ - consistent with Samson et al. 2006 to 2012 rate (40.95\%) (19)—but has significant survival benefit, indicating a powerful opportunity to extend the lives of esophageal cancer patients. It is promising, however, that we show an increase in odds of adherence from 2006 to 2016 when compared to the group in 2004 to 2005. A study on the NCDB evaluating nodal staging showed a similar increasing trend in adequate nodal staging (52.6\% in 2015 vs. $26.0 \%$ in 2004) over time (27). Future studies should investigate the barriers to achieving this life-extending quality measure. A possible reason behind this perceived deficit in quality is lymph nodes' tendency to break into pieces in the operating room. No measure exists in the NCDB to evaluate whether whole or parts of lymph nodes were removed. We do not know if hospitals are counting whole lymph nodes or parts. If parts are being counted as whole, it is possible that we have overestimated the adherence rate; if parts are being omitted from the lymph node count, then it is possible that adherence is much higher than we have calculated.

Like the other quality measures, nodal staging was developed from accepted guidelines. The NCCN guidelines cite Rizk et al. who stratify their recommendations by $\mathrm{T}$ classification $\mathrm{T} 1$ to $\mathrm{T} 3$ ranging from 10 to 30 lymph nodes resected in patients who only undergo esophagectomy (28). The NCCN simplifies this recommendation requiring resection of at least 15 lymph nodes but declines to make a recommendation on those who receive chemotherapy and/or radiation and esophagectomy (11). We amended this original guideline to include any patient that had an operation on the chest. Despite this adjustment, we showed survival benefit for patients who had adequate lymph node resection, affirming this legitimacy and usefulness of this quality measure.

Completeness of resection is unique in that it is the only quality measure that evaluates an outcome. No guideline explicitly states that resection should be to R0, presumably because guidelines tend to describe processes and not outcomes after the action of care is complete. Nonetheless, it has been shown that positive margins lead to worse outcomes in patients receiving esophagectomy (29). Our study concurs in that $\mathrm{R} 0$ resection was most predictive of increasing time to all-cause mortality of all four quality measures. Fortunately, most patients do receive an R0 resection nationally. Strong adherence to this quality measure should be maintained.

\section{Demographic correlations}

Advanced age, Medicaid insurance status, and Black ethnicity were all associated with statistically significant decreased odds of adherence in one or more of the measures. These associations may be explained by factors not explored by this study. These include disturbances in treatment planning and execution due to patients lost to follow up or provider bias. On the other hand, adherence improved in the more recent period, with cases after 2010 being associated with increased odds of adherence in administration of induction therapy, adequate nodal staging, and completeness of resection. The trend of increasing adherence to induction therapy may be explain by the date of publication of the CROSS trial (in 2012), the landmark study that showed improved survival in patients with induction chemotherapy and radiation and then surgery (12). Similarly, the study from which our nodal staging quality measure was derived was published in 2010 (28). In other words, we should expect that standardized best practices gain popularity among providers once is evidence and consensus builds.

\section{Survival improved as the number of quality measures adbered to increased}

Patients whose care adhered to two of four quality measures had significantly increased time to all-cause mortality when compared to that of patients with care adherent to one of four quality measures. The same is true when comparing the group with three of four quality measures adhered to the group with two of four. The incremental increase in survival, however, decreases every time. These results suggest that as the number of quality measures adhered to 


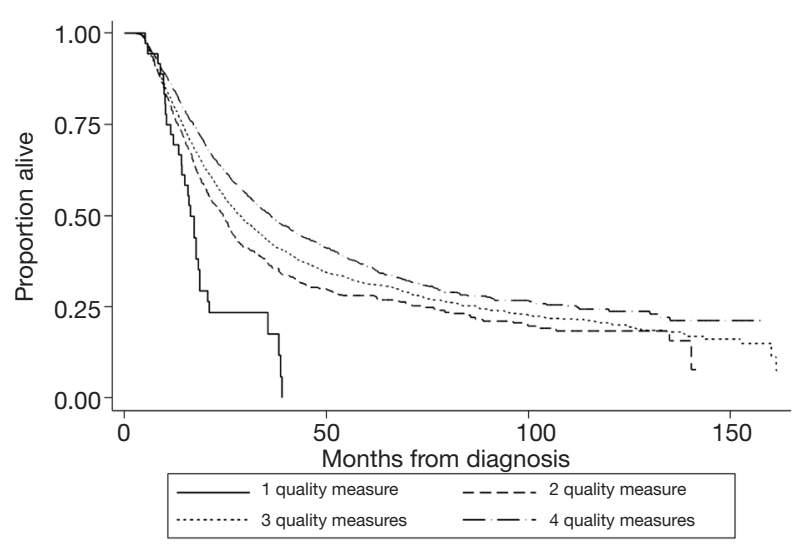

Figure 3 Kaplan-Meier survival analysis showing the proportion of survival over time for those that were adherent to one of four, two of four, three of four, and four of four quality measures $(\mathrm{P}<0.001)$.

increases, survival increases. Samson et al. showed similarly for 2006 to 2012 data that overall survival increased as number of quality measures adhered to increased (19). In theory, a patient's time to death would increase infinitely as quality measures are progressively added, albeit with smaller increments each time (Figure 3).

It would, however, be progressively more difficult to identify novel quality measures as the more convenient data fields would have already been evaluated. Furthermore, factors affecting the patient's all-cause mortality other than their primary disease-comorbid disease, trauma-not captured by the NCDB would progressively accumulate and decrease survival benefit.

\section{Limitations}

This study provides insight into baseline adherence to best practices nationally in the care of esophageal cancer patients; yet, it fails to characterize causal factors that contribute to increased or decreased adherence. Associations may be made between certain patient characteristics and adherence as discussed, however, but causality is beyond the scope of this current work. This study is also limited in its ability to comment of structural quality of patient care and its association with outcomes.

Furthermore, while these quality measures attempt to capture the most salient points of best practice today and are derived from standard guidelines and landmark studies, they are not formally published themselves. We assume that providers are aware of these practices in the given period of 2004 to 2016. Indeed, many of the studies and guidelines used to develop these quality measures were published after 2004, which means we are scrutinizing a patient's care with best practices published after their diagnosis. For that reason, this study does not reflect implementation and acceptance of best practices nationally, but rather a baseline evaluation of the state of care.

This study is further limited by the characteristics of the NCDB, which captures only $70 \%$ of cancer diagnoses. Data is only included if it is reported to the NCDB, which is only required of $\mathrm{CoC}$-accredited hospitals. There are aspects of care beyond management that affect adherence to care, such as demographics, socioeconomic factors, and patient choice. While we controlled for what we could in the NCDB, we are limited by the fields present. We certainly do not capture the whole picture with this study. With respect to our quality measures, lack of fields needed to calculate the timing of surgery after chemotherapy and the size and fraction of lymph nodes collected limit precision of this analysis. Moreover, while the AJCC did publish its 7 th edition of staging criteria in 2009 (the middle of our selected period of 2004 to 2016), we do not believe that this introduced inconsistency in patient inclusion in this study because only subclassifications within stage III changed. In other words, only those deemed stage III by the $6^{\text {th }}$ edition would be deemed stage III by the $7^{\text {th }}$ edition. Finally, while there are certain fields were only required after a certain year in the NCDB, those were not used in this study.

\section{Future projects}

As data in the NCDB becomes more current and best practices become more well-known, this analysis should be repeated. Future projects could investigate the reasons for variability in quality measure adherence. They could also investigate why certain demographic characteristics predict increased or decreased likelihood of high-quality care. Structural aspects of quality still need to be evaluated as well. Finally, while we were unable to performed a cost-effectiveness analysis for implementing these quality measures due to limitations in the NCDB fields, it should be performed on a different dataset or at an individual hospital as cost is potentially a major inhibitor to implementing these life extending practices.

\section{Conclusions}

There is adequate national adherence to three out of the four quality measures that were identified. Significant 
opportunity exists to extend the life of esophageal cancer patients if the barriers to adequate nodal staging are investigated, identified, and ultimately conquered. Adherence to induction therapy and completeness of resection is strong and should be maintained because there is significant survival benefit. This study shows that survival improves as the number of quality measures adhered to improves. While this improvement is incrementally smaller and probably limited by the identification of effective quality measures, it provides direction for future quality improvement and research projects.

\section{Acknowledgments}

Kathryn Engelhardt, MD assisted in data analysis.

Funding: D Odell reports grants from American Cancer Society Grant (K07 CA216330) during the conduct of the study.

\section{Footnote}

Reporting Checklist: The authors have completed the STROBE reporting checklist. Available at http://dx.doi. org/10.21037/jtd-20-1347

Conflicts of Interest: All authors have completed the ICMJE uniform disclosure form (available at http://dx.doi. org/10.21037/jtd-20-1347). DO reports grants from American Cancer Society Grant (K07 CA216330) during the conduct of the study. The authors have no other conflicts of interest to declare.

Ethical Statement: The authors are accountable for all aspects of the work in ensuring that questions related to the accuracy or integrity of any part of the work are appropriately investigated and resolved. The study was conducted in accordance with the Declaration of Helsinki (as revised in 2013). This study of deidentified, public data was ruled exempt by the Northwestern University Institutional Review Board and informed consent was not required as it is defined by the United States Department of Health and Human Services as secondary research of biospecimens that are publicly available.

Open Access Statement: This is an Open Access article distributed in accordance with the Creative Commons Attribution-NonCommercial-NoDerivs 4.0 International License (CC BY-NC-ND 4.0), which permits the non- commercial replication and distribution of the article with the strict proviso that no changes or edits are made and the original work is properly cited (including links to both the formal publication through the relevant DOI and the license). See: https://creativecommons.org/licenses/by-nc-nd/4.0/.

\section{References}

1. National Cancer Institute. Esophageal Cancer Cancer Stat Facts 2019. Available online: https://seer.cancer.gov/ statfacts/html/esoph.html

2. Berry MF. Esophageal cancer: Staging system and guidelines for staging and treatment. J Thorac Dis 2014;6 Suppl 3:S289-97.

3. Varghese TK, Hofstetter WL, Rizk NP, et al. The society of thoracic surgeons guidelines on the diagnosis and staging of patients with esophageal cancer. Ann Thorac Surg 2013;96:346-56.

4. Enzinger PC, Mayer RJ. Esophageal cancer. N Engl J Med 2003;349:2241-52.

5. Bruzzi J, Marom EM, Hofstetter WL, et al. Esophageal Cancer. Oncologic Imaging: A Multidisciplinary Approach. Philadelphia, PA: Elsevier Inc., 2012;211-30.

6. Evans JA, Early DS, Chandraskhara V, et al. The role of endoscopy in the assessment and treatment of esophageal cancer. Gastrointest Endosc 2013;77:328-34.

7. Lordick F, Mariette C, Haustermans K, et al. Oesophageal cancer: ESMO clinical practice guidelines for diagnosis, treatment and follow-up. Ann Oncol 2016;27:v50-7.

8. Little AG, Lerut AE, Harpole DH, et al. The Society of Thoracic Surgeons practice guidelines on the role of multimodality treatment for cancer of the esophagus and gastroesophageal junction. Ann Thorac Surg 2014;98:1880-5.

9. Malthaner RA, Wong RKS, Rumble RB, et al. Preoperative or postoperative therapy for resectable esophageal cancer: guideline recommendations. Toronto $(\mathrm{ON})$ : Cancer Care Ontario, 2008. Program in Evidencebased Care Evidence-based Series No. 2-11 Version 4. Endorse 2008.

10. Esophageal Cancer Guidelines 2017. Available online: http://misc.medscape.com/pi/iphone/medscapeapp/html/ A278744-business.html

11. Ajani JA, Barthel JS, Bentrem DJ, et al. Esophageal and esophagogastric junction cancers. J Natl Compr Canc Netw 2011;9:830-87.

12. van Hagen P, Hulshof MCCM, van Lanschot JJB, et al. Preoperative Chemoradiotherapy for Esophageal or 
Junctional Cancer. N Engl J Med 2012;366:2074-84.

13. Franko J, Voynov G, Goldman CD. Esophagectomy timing after neoadjuvant therapy for distal esophageal adenocarcinoma. Ann Thorac Surg 2016;101:1123-30.

14. Ferron G, Martinez A, Gladieff L, et al. Adherence to guidelines in gynecologic cancer surgery. Int J Gynecol Cancer 2014;24:1675-8.

15. Lin HY, Bedrosian I, Babiera G V., et al. Using the National Cancer Data Base for quality evaluation to assess adherence to treatment guidelines for nonmetastatic inflammatory breast cancer. Cancer 2017;123:2618-25.

16. Graboyes EM, Garrett-Mayer E, Sharma AK, et al. Adherence to National Comprehensive Cancer Network guidelines for time to initiation of postoperative radiation therapy for patients with head and neck cancer. Cancer 2017;123:2651-60.

17. Charlton ME, Mattingly-Wells LR, Marcet JE, et al. Association between surgeon characteristics and their preferences for guideline-concordant staging and treatment for rectal cancer. Am J Surg 2014;208:817-23.

18. Minami CA, Wayne JD, Yang AD, et al. National Evaluation of Hospital Performance on the New Commission on Cancer Melanoma Quality Measures. Ann Surg Oncol 2016;23:3548-57.

19. Samson P, Puri V, Broderick S, et al. Adhering to Quality Measures in Esophagectomy Is Associated With Improved Survival in All Stages of Esophageal Cancer. Ann Thorac Surg 2017;103:1101-8.

20. American College of Surgeons. Participant User Files. National Cancer Data Base - Data Dictionary PUF 2014 2020. Available online: http://ncdbpuf.facs.org/About

21. US Department of Health and Human Services. Revised Common Rule. Available online: https://www.hhs.gov/

Cite this article as: Adhia A, Feinglass J, Schlick CJ, Odell D. Adherence to quality measures improves survival in esophageal cancer in a retrospective cohort study of the national cancer database from 2004 to 2016. J Thorac Dis 2020;12(10):5446-5459. doi: $10.21037 /$ jtd-20-1347 ohrp/regulations-and-policy/regulations/finalizedrevisions-common-rule/index.html

22. Donabedian A. The Milbank Memorial Fund. Science 1923;58:437.

23. Tessier W, Gronnier C, Messager M, et al. Does timing of surgical procedure after neoadjuvant chemoradiation affect outcomes in esophageal cancer? Ann Thorac Surg 2014;97:1181-9.

24. Depypere L. The effect of time interval on esophagectomy after neoadjuvant treatment. Ann Transl Med 2016;4:117.

25. Ruol A, Rizzetto C, Castoro C, et al. Interval between neoadjuvant chemoradiotherapy and surgery for squamous cell carcinoma of the thoracic esophagus: Does delayed surgery have an impact on outcome? Ann Surg 2010;252:788-96.

26. Chiu $\mathrm{CH}$, Chao YK, Chang HK, et al. Interval Between Neoadjuvant Chemoradiotherapy and Surgery for Esophageal Squamous Cell Carcinoma: Does Delayed Surgery Impact Outcome? Ann Surg Oncol 2013;20:4245-51.

27. Schlick CJR, Khorfan R, Odell DD, et al. Adequate Lymphadenectomy as a Quality Measure in Esophageal Cancer: Is there an Association with Treatment Approach? Ann Surg Oncol 2020;27:4443-56.

28. Rizk NP, Ishwaran H, Rice TW, et al. Optimum lymphadenectomy for esophageal cancer. Ann Surg 2010;251:46-50.

29. Javidfar J, Speicher PJ, Hartwig MG, et al. Impact of positive margins on survival in patients undergoing esophagogastrectomy for esophageal cancer presented at the poster session of the fifty-first annual meeting of the society of Thoracic Surgeons, San Diego, CA, Jan 24-28, 2015. Ann Thorac Surg 2016;101:1060-7. 